\title{
Phase Separation in Poly( $N$-isopropyl acrylamide)/Water Solutions I. Cloud Point Curves and Microgelation
}

\author{
Xu Zheng, Zhen Tong, ${ }^{\dagger}$ Xiaoli XIE, and Fang Zeng \\ Research Institute of Materials Science, South China University of Technology, \\ Guangzhou 510641, People's Republic of China
}

(Received August 7, 1997)

\begin{abstract}
Cloud point curve was determined on binary and ternary aqueous solutions of several fractionated poly $(N$ isopropyl acrylamide) (PNIPA) samples with the molecular weight $M_{\eta}=11.3 \times 10^{4}-210 \times 10^{4}$ and the molecular weight distribution $M_{w} / M_{n}=1.21-1.62$. In both the binary and ternary solutions, the cloud point temperature $T_{\text {clo }}$ slightly increases with the increase in molecular weight of PNIPA. The concentration dependence for the binary solution is weak while it becomes obvious for the ternary solution containing two different PNIPA samples in water; i.e., the molecular weight distribution alters the shape of the cloud point curve. The storage and loss moduli $G^{\prime}$ and $G^{\prime \prime}$ for the PNIPA sample of $M_{\eta}=210 \times 10^{4}$ in water at concentrations $2.2-8.5 \mathrm{wt} \%$ were measured at several temperatures encompassing the $T_{\text {clo. }}$. The results indicate that the solution changes from a homogeneous fluid into viscoelastic microgels suspended in a liquid phase during the phase separation. This physical network is considered to be jointed via the intermolecular hydrophobic bonding between the $N$-isopropyl groups, which may provide a model system for the structure change of proteins induced in the denaturation.

KEY WORDS Poly $(N$-isopropyl acrylamide) / Cloud Point Curve / Microgelation / Hydrophobic Bonding / Physical Crosslink / Sol-Gel Transition / Dynamic Viscoelasticity /
\end{abstract}

Poly( $N$-isopropyl acrylamide) (PNIPA) becomes an attractive polymeric material academically and industrially due to the temperature sensitivity found in its aqueous solutions and hydrogels. ${ }^{1}$ It is known that the PNIPA/water solution undergoes phase separation upon heating, exhibiting a lower critical solution temperature (LCST) at about $32^{\circ} \mathrm{C}^{2}$ and the interaction leading to the phase separation is considered as the hydrophobic bonding among the alkane side chains on the polymer. In contrast to this system, most polymer solutions showing the LCST involve a free volume change during the phase separation. ${ }^{3,4}$

A large amount of papers have been published concerning the phase separation of the PNIPA/water solution in the last decade. ${ }^{1}$ The coil-to-globule transition of PNIPA single chain was found in dilute aqueous solutions just below its LCST by dynamic light scattering with elevating temperature. ${ }^{5-8}$ The aggregation of PNIPA chains during the phase separation was observed using fluorescence technique. ${ }^{9-12}$ Differential scanning calorimetry (DSC) measurements ${ }^{13-15}$ illustrated an endotherm at the separation temperature of the PNIPA/ water solution, indicating the elimination of polymerwater contacts with increasing temperature.

In spite of the importance to understanding the mechanism of phase separation, the information about the phase diagram of the PNIPA/water solution is still confused, sepecially for the concentration and molecular weight dependence of the cloud point temperature $T_{\text {clo. }}$. Heskins and Guillet ${ }^{13}$ reported a cloud point curve with the LCST at its minimum about $15 \mathrm{wt} \%$ by visible observation on an unfractionated PNIPA sample. Fujishige $e t a l .{ }^{6}$ argued that the cloud points of PNIPA/ water solutions were independent of either solution concentration or molecular weight according to their optical transmittance. By DSC measurement, Schild and Tirrell ${ }^{14}$ showed that the cloud point for PNIPA in water

\footnotetext{
${ }^{\dagger}$ To whom correspondence should be addressed
}

depended on polymer molecular weight. However, there were some deficiencies in the above experiment procedures, which would be discussed later. Up to now, neither the critical concentration nor binodals is reported for this system though it is usually considered having the LCST.

Recently, we studied the phase separation process of the PNIPA/water solution by NMR spectra at several temperatures encompassing the $T_{\text {clo }}{ }^{16}$ The change in the spin-lattice relaxation time $T_{1}$ with temperature for different protons indicates that the relaxation of the $\mathrm{N}$ isopropyl side chain slows down while that of the main chain speeds up due to the appearance of heterogeneity in the solution. This phenomenon suggests that during the phase separation the ordered water molecules surrounding hydrophobic $N$-isopropyl groups are dissociated from the hydrated shell and the hydrophobic bonding is formed among these groups. The latter results in restriction on the motion of the methyl group in the $N$-isopropyl group of PNIPA after phase separation. When the solution is concentrated enough, the hydrophobic bonding may form among the $N$-isopropyl groups attaching to different PNIPA chains, which causes a network structure in the PNIPA/water solution during the phase separation. Our dynamic viscoelasticity experiments ${ }^{17}$ confirmed the appearance of this reversible network formed by the hydrophobic bonding in a 1.22 wt $\%$ aqueous solution of PNIPA with viscosity-averaged molecular weight of $210 \times 10^{4}$, though no well-shaped macroscopic gel was obtained after phase separation.

Above findings suggest that the phase separation observed from the PNIPA/water solution would be the manifestation of the physically crosslinking process upon heating. The solution, however, becomes heterogeneously milk-like after phase separation and no macroscopic phase of the physical gel can be separated from the solution, leading to a new catalogue of phase separation and gelation. This paper presents the cloud point curves of binary and ternary PNIPA/water solu- 
tions to reveal the effects of the molecular weight and molecular weight distribution. The process of network formation upon heating has also been followed by dynamic viscoelasticity measurements.

\section{EXPERIMENTAL}

\section{Sample Preparation}

$N$-Isopropyl acrylamide (NIPA) monomer provided by Cohjin Co. was recrystallized twice from a benzene/ $n$-hexane mixture prior to use. The polymerization was conducted in tert-butyl alcohol at $55^{\circ} \mathrm{C}$ for $8 \mathrm{~h}$ with the monomer concentration of $1.073 \mathrm{~mol} \mathrm{~L}^{-1}$ and the initiator azobis(isobutylonitrile) of $0.58 \mathrm{~mol} \%$. The obtained PNIPA sample was purified by precipitation three times from acetone solution into $n$-hexane and then fractionated by phase separation in acetone $/ n$-hexane mixtures at fixed temperature for several times. ${ }^{18}$ Six fractions with reasonably narrow molecular weight distribution were chosen to be used in this study.

The intrinsic viscosity $[\eta]$ of PNIPA samples was measured in methanol at $25^{\circ} \mathrm{C} \pm 0.05^{\circ} \mathrm{C}$ with a modified Ubbelohde viscometer. The viscosity average molecular weight $M_{\eta}$ of the sample was evaluated from [ $\left.\eta\right]$ using the Mark-Houwink-Sakurada equation created by Chiantore et al. ${ }^{19}$ The index of molecular weight distribution $M_{w} / M_{n}$ was determined by a Waters-150C gel permeation chromatography (GPC) with three $\mu$-Styragel columns, using narrowly distributed polystyrene as the standards. The characterization data of all the PNIPA samples are listed in Table I.

\section{Cloud Point Determination}

A dried PNIPA sample was dissolved in doubly distilled water to make a stock binary solution at the concentration $C \sim 12 \mathrm{wt} \%$. The solution was divided into several parts by pouring it into cylindrical cells and diluted with proper amount of water. The cell was then sealed for cloud point measurement. Ternary PNIPA/ water solutions were made in the similar procedure except that two different PNIPA samples were dissolved in water to make the stock ternary solution. The PNIPA samples used in the ternary solution were $\mathrm{T} 1$ and $\mathrm{T} 2$, and the weight fraction $\xi$ of $\mathrm{T} 1$ in the mixtures coded MT-70 and MT-30 was 0.70 and 0.30 , respectively. The $M_{w}$ and $M_{w} / M_{n}$ data of the mixtures evaluated from the component values are also listed in Table I. Figure 1 depicts the GPC curves of the PNIPA mixtures MT-70 and MT-30. The two peaks represent the component PNIPA samples T1 and T2 in each mixture.

Cloud points were determined by monitoring the transmittance of a $\mathrm{He}-\mathrm{Ne}$ laser beam passing through the solution as a function of temperature. ${ }^{6,20}$ The cell containing a test solution was mounted in a thermostatically controlled water bath and the temperature was increased or decreased at the rate about $2^{\circ} \mathrm{Ch}^{-1}$ near the cloud point. The intensity of the transmitted light decreased sharply when temperature passed through the cloud point.

\section{Dynamic Modulus Measurement}

Dynamic moduli were measured on aqueous solutions of the sample D1 at 5 concentrations ranging $2.21-$
Table I. Molecular characteristics of $\operatorname{poly}(N$ isopropyl acrylamide) samples

\begin{tabular}{ccc}
\hline Sample code & $10^{-4} M_{\eta}$ & $M_{w} / M_{n}{ }^{\mathrm{a}}$ \\
\hline D1 & 210 & 1.62 \\
D2 & 121 & 1.21 \\
D3 & 39 & 1.33 \\
D4 & 18 & 1.39 \\
T1 & 57.8 & 1.45 \\
T2 & 11.3 & 1.26 \\
$-------------1.69^{\mathrm{c}}$ \\
MT-70 $(\xi=0.70)$ & $43.9^{\mathrm{b}}$ & $1.70^{\mathrm{c}}$ \\
MT-30 $(\xi=0.30)$ & $25.3^{\mathrm{b}}$ &
\end{tabular}

${ }^{\text {a }}$ Measured by GPC using narrowly distributed polystyrene as the standard. ${ }^{\mathrm{b}}$ Calculated $M_{w}$ from the $M_{\eta}$ values of T1 and T2 with the given composition $\xi$. ${ }^{\mathrm{c}}$ Calculated from the $M_{w}$ and $M_{n}$.
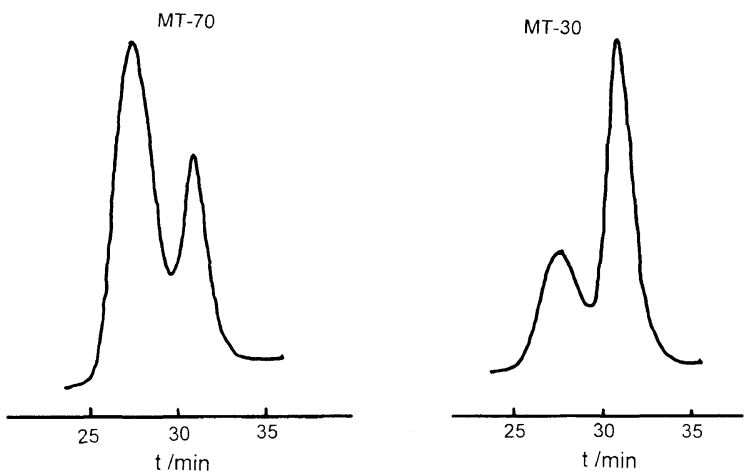

Figure 1. GPC curves for the PNIPA mixtures MT-70 and MT-30; the peak on the left is $\mathrm{T} 1$ and the right is $\mathrm{T} 2$.

$8.53 \mathrm{wt} \%$ with a strain-controlled rheometer RFS-II of Rheometrics. ${ }^{17}$ The Couette fixture was used whose cup and bob diameters were 33.96 and $32 \mathrm{~mm}$, respectively, and bob length was $33.31 \mathrm{~mm}$. The temperature was controlled by a computer programmable circulator in the range 25 to $35^{\circ} \mathrm{C}$ with the precision of $\pm 0.1^{\circ} \mathrm{C}$. After confirming the linear viscoelasticity region at different temperatures, the applied oscillatory strain was chosen at $10-20 \%$ to produce enough torque within the linear region.

\section{RESULTS AND DISCUSSION}

\section{Cloud Point Curves}

Two typical curves of transmittance against temperature are shown in Figure 2 for the ternary solutions of the mixture MT-70 in water at concentrations of 7.10 and $0.52 \mathrm{wt} \%$ during heating. Their cloud point temperatures can be undoubtedly determined from the break point of the corresponding transmittance curves as 32.9 and $33.4^{\circ} \mathrm{C}$. The transmittance curve during cooling process almost coincides with that in the heating process and no retardation of the appearance of phase separation is observed in cooling process. All cloud points in this work were determined from the heating curves. The detectable retardation between heating and cooling was reported by Fujishige et $a l^{6}$ as their heating rate of $1^{\circ} \mathrm{C} \min ^{-1}$ was too high.

Figure 3 shows the cloud point data for the binary PNIPA/water solutions. It is seen that all the cloud point curves are very flat, and the cloud point temperature $T_{\text {clo }}$ at a given concentration increases with the molecular 


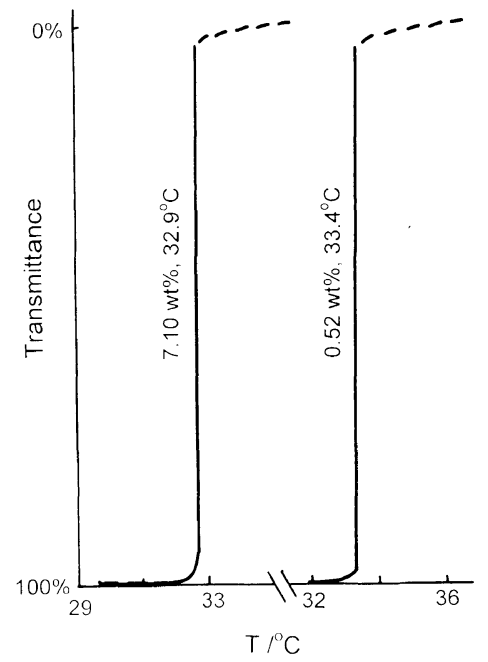

Figure 2. Temperature dependence of the transmittance of MT$70 /$ water solutions with concentrations 7.10 (left) and $0.52 \mathrm{wt} \%$ (right) on heating.

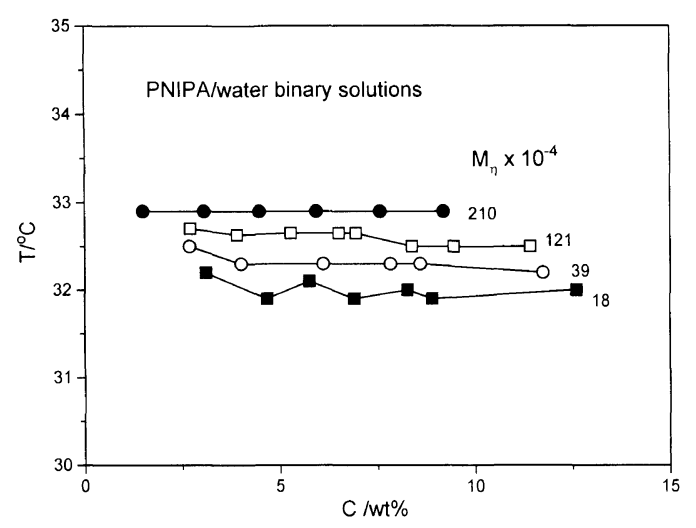

Figure 3. Cloud points of binary PNIPA/water solutions.

weight of PNIPA samples. For an example, the $T_{\text {clo }}$ for the sample D4 with the lowest molecular weight of $M_{\eta}=18 \times 10^{4}$ is about $32^{\circ} \mathrm{C}$, while that for the sample Dl with the highest $M_{\eta}=210 \times 10^{4}$ is $32.9^{\circ} \mathrm{C}$. The concentration dependence becomes observable when the molecular weight of PNIPA decreases. This tendency can be recognized from the cloud point curves of D2, D3, and D4 in water solutions. Nevertheless, the maximum variation in $T_{\text {clo }}$ is within $0.3^{\circ} \mathrm{C}$ for all examined binary PNIPA/water solutions.

To investigate the molecular weight and concentration dependence further, we determined the cloud point curve for the aqueous solution of PNIPA mixtures MT-70 and MT-30, and plotted them in Figure 4. The concentration dependence of cloud temperature for the ternary solution looks obvious than that in Figure 3 for the binary solutions, especially in the dilute region where $T_{\text {clo }}$ rises up with decreasing concentration. If the components $\mathrm{T} 1$ and $\mathrm{T} 2$ in the mixtures are roughly regarded as monodisperse, the weight-averaged molecular weights $M_{w}$ evaluated for MT-70 and MT-30 are $43.9 \times 10^{4}$ and $25.3 \times 10^{4}$ (Table I), respectively. It is seen that the cloud point curves of these ternary PNIPA/water solutions are still located following the rule established from the binary system, i.e., the $T_{\text {clo }}$ moves upward as the $M_{w}$ of the NIPA sample becomes higher. Though the cloud points of the MT-30 solutions are above those for the D3

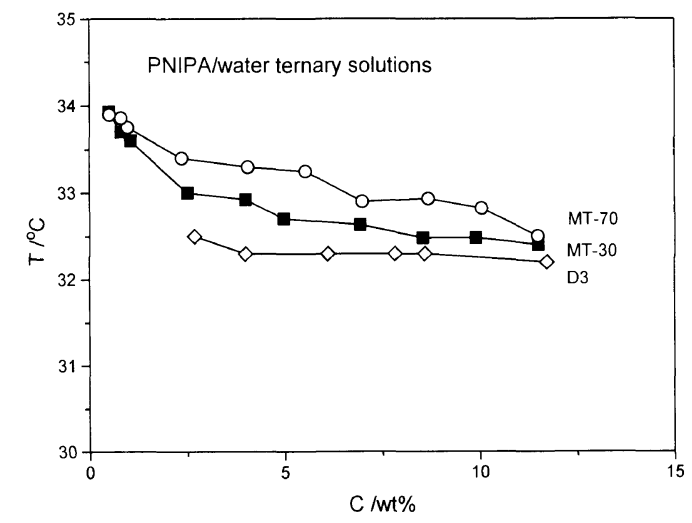

Figure 4. Cloud points of ternary solutions of PNIPA mixtures MT-70 and MT-30 in water compared with those of the binary D3/water solution.

solutions, their molecular weights are incomparable due to the completely different distributions (see Figure 1). This fact indicates again that the molecular weight distribution affects the shape of the cloud point curve considerably. ${ }^{21}$

Summarizing the above results, we can obtain two features in the phase separation behavior of the PNIPA/ water solution. First, the cloud point temperature, $T_{\text {clo }}$ is somewhat dependent on the solution concentration in case of a narrowly distributed PNIPA in water. This dependency becomes obvious if the molecular weight is decreased or its distribution is broadened. Second, there is a molecular weight dependence of the cloud point curve for the PNIPA/water solution. For the solution having higher molecular weight PNIPA, the cloud point curve appears at higher temperature. The detail cloud temperature is affected by the molecular weight distribution of the PNIPA sample. In contrast, for most polymer solutions with the LCST $T_{\text {clo }}$ becomes lower as the molecular weight of polymer increases. ${ }^{3,4,21}$ Typical thermodynamic theory for polymer solutions with the LCST also predicts the decrease of $T_{\text {clo }}$ with increasing polymer molecular weight. ${ }^{22,23}$ The observed abnormal behavior of molecular weight dependence of cloud point curves intimates a different mechanism for the phase separation in this system, which will be discussed in the following section.

The confusion in reported phase separation behavior may be arisen from the experimental procedure. Heskins and Guillet $^{13}$ measured cloud point curve on one unfractionated PNIPA sample, showing an obvious minimum at about $15 \mathrm{wt} \%$ with a extremely sharp $T_{\text {clo }}$ increase in very dilute region and a gradual $T_{\text {clo }}$ rise in the concentrated region (even higher than $30 \mathrm{wt} \%$ ). While their visual observation method would face difficulty in determining the appearance of turbidity in very dilute PNIPA/water solutions. Fujishige et al. ${ }^{6}$ found the independence of the cloud point on either solution concentration or molecular weight in the dilute region of $0.033-1.0 \mathrm{wt} \%$. But their rate of temperature change is too fast $\left(1{ }^{\circ} \mathrm{Cmin}^{-1}\right)$ to observe these delicate dependencies. By DSC measurement, Schild and Tirrell ${ }^{14}$ argued that the cloud point for PNIPA in water depended on polymer molecular weight. However, the samples they used were widely distributed $\left(M_{w} / M_{n}>6.0\right)$ and only one extremely dilute solution of $0.04 \mathrm{wt} \%$ was tested for each 


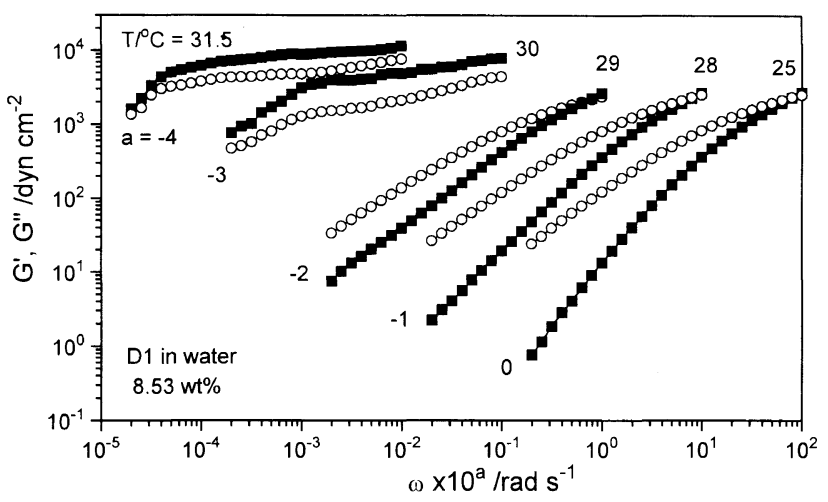

Figure 5. Angular frequency $\omega$ dependence of storage and loss moduli $G^{\prime}$ (solid square) and $G^{\prime \prime}$ (open circle) of the D1/water solution with $C=8.53 \mathrm{wt} \%$ at indicated temperatures. The data are shifted to avoid overlapping by $10^{\mathrm{a}}$ as indicated in the figure.

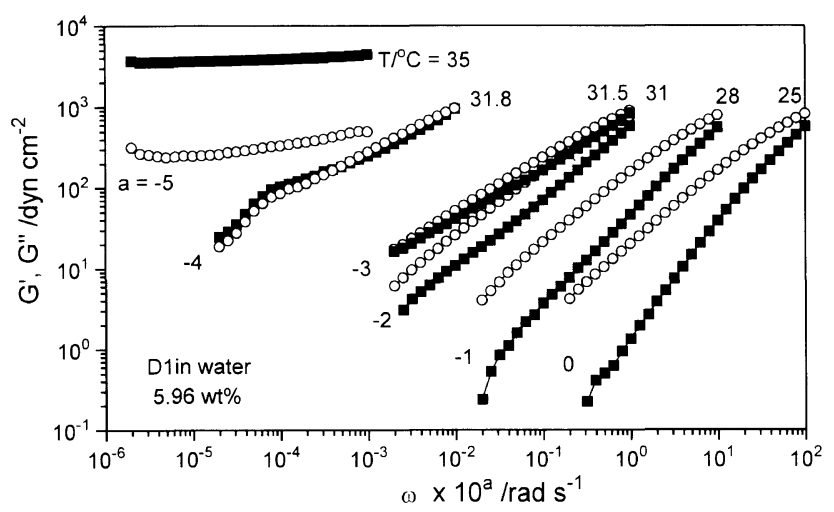

Figure 6. Angular frequency $\omega$ dependence of storage and loss moduli $G^{\prime}$ (solid square) and $G^{\prime \prime}$ (open circule) of the D1/water solution with $C=5.96 \mathrm{wt} \%$ at indicated temperatures. The data are shifted to avoid overlapping by $10^{\mathrm{a}}$ as indicated in the figure.

sample. Their cloud point temperature scatters from $32.2^{\circ} \mathrm{C}$ to $35.7^{\circ} \mathrm{C}$ for different samples.

\section{Microgelation}

Figures 5 through 9 show the angular frequency $\omega$ dependence of the storage and loss moduli $G^{\prime}$ and $G^{\prime \prime}$ for the D1/water solutions of 5 concentrations at different temperatures. The data are shifted along the abscissa by a factor of $10^{\mathrm{a}}$ in order to avoid overlapping. The contribution of solvent viscosity to the loss modulus is below 5 per mill and neglected here. At lower temperatures, $G^{\prime}$ and $G^{\prime \prime}$ in the low $\omega$ region are proportional to $\omega^{1.5-1.8}$ and $\omega^{1}$, respectively; which is the characteristic of a viscous liquid. ${ }^{24}$ Broad molecular weight distribution of the D1 sample may prevent us achieving a theoretical low-frequency limit of $\omega^{2}$ for the $G^{\prime}$. With increasing frequency, both the $G^{\prime}$ and $G^{\prime \prime}$ increase monotonically without the sign of appearance of the plateau zone in observed $\omega$ region and the slope of the $G^{\prime}$ and $G^{\prime \prime}$ curves is still larger than theoretically predicted $0.67 .{ }^{24}$ The above dynamic mechanical properties indicate that the PNIPA/water solution remains a homogeneous fluid at these lower temperatures.

At higher temperatures, $G^{\prime}$ becomes larger than $G^{\prime \prime}$ and there appears a plateau in the $G^{\prime} v s$. $\omega$ curve over the whole $\omega$ region observed for all concentrations. The $G^{\prime \prime}$ approaches to parallel with $G^{\prime}$ at certain temperatures

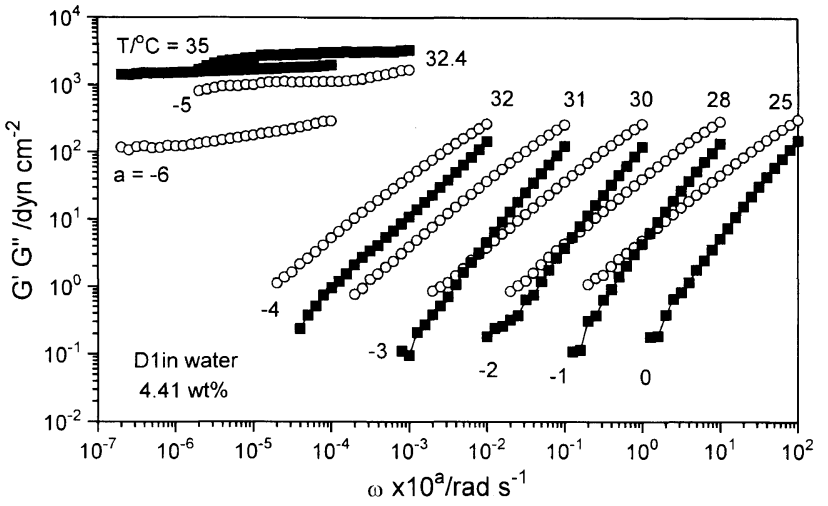

Figure 7. Angular frequency $\omega$ dependence of storage and loss moduli $G^{\prime}$ (solid square) and $G^{\prime \prime}$ (open circle) of the D1/water solution with $C=4.41 \mathrm{wt} \%$ at indicated temperatures. The data are shifted to avoid overlapping by $10^{\mathrm{a}}$ as indicated in the figure.

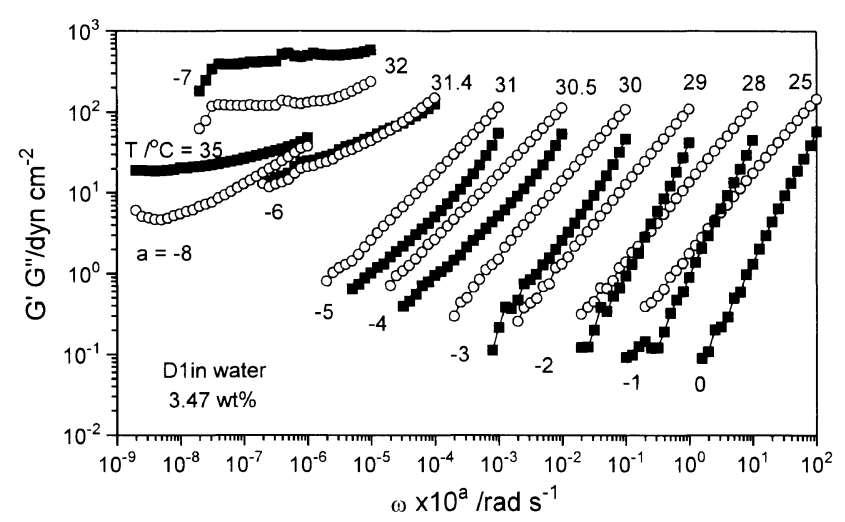

Figure 8. Angular frequency $\omega$ dependence of storage as loss moduli $G^{\prime}$ (solid square) and $G^{\prime \prime}$ (open circle) of the D1/water solution with $C=3.47 \mathrm{wt} \%$ at indicated temperatures. The data are shifted to avoid overlapping by $10^{\mathrm{a}}$ as indicated in the figure.

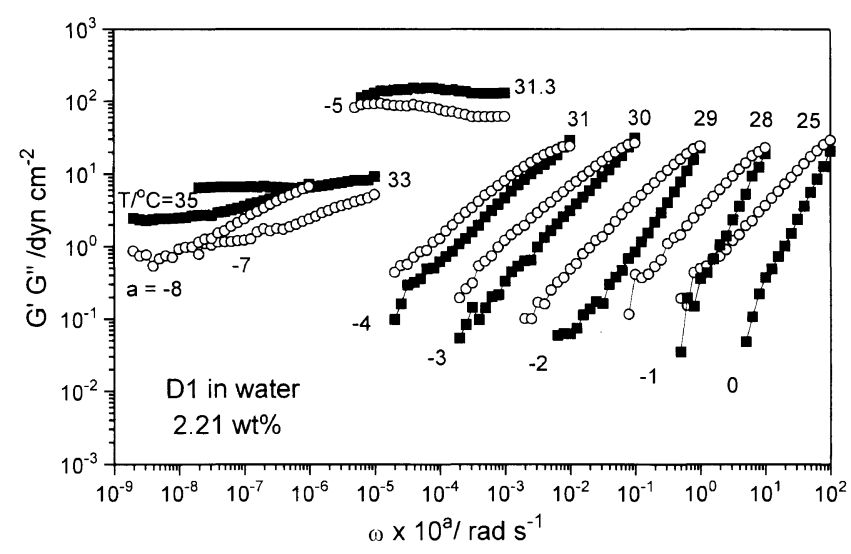

Figure 9. Angular frequency $\omega$ dependence of storage and loss moduli $G^{\prime}$ (solid square) and $G^{\prime \prime}$ (open circle) of the Dl/water solution with $C=2.21 \mathrm{wt} \%$ at indicated temperatures. The data are shifted to avoid overlapping by $10^{\mathrm{a}}$ as indicated in the figure.

(saying $31.5^{\circ} \mathrm{C}$ for $5.96 \mathrm{wt} \%$ solution in Figure 6). This phenomenon demonstrates that the PNIPA chains in aqueous solution at these temperatures associate with each other to form a crosslinked network characterized by the equilibrium modulus $G_{\text {eq }}$. The network formation in the PNIPA/water solution is reversible when the solution is cooled down and reproducible when the cooled solution is heated again. Other evidence for the 
network formation is that a aging time is required in DSC measurement of the PNIPA/water solution after cooled from phase separated state to homogeneous one, ${ }^{25}$ which is for the PNIPA molecules to recover their hydrated structure with water molecules. We consider that the hydrophobic bonding among $N$-isopropyl groups on the PNIPA side chains acts as the physical joint in this process. ${ }^{16,17}$ The life time of the hydrophobic bonding crosslinks is so long that they cannot relax at the tested low $\omega$ end.

The size of the physical gels in the PNIPA/water solutions seems to be in the order of $\mu \mathrm{m}$ since after phase separation the solution becomes heterogeneously milklike and no macroscopic gel phase can be separated from the solution. Therefore, what we have observed during the phase separation is just that the PNIPA/water solution changes from a homogeneous viscous liquid to a suspension of microscopic viscoelastic gels dispersed in water or in an extremely dilute solution. In other words, this phase separation should be virtually the sol-gel transition, referred to as microgelation. The driving force for this microgelation is the hydrophobic interaction which is a cooperating interaction of the hydrogen bonds and van der Waals force. When PNIPA molecules are dispersed in water, water molecules form a hydrated shell of ordered ice-like structure upon the hydrogen bonds between water molecules themselves around the hydrophobic part of the polymer, making this polymer water-soluble. ${ }^{16}$ This hydrated shell will be partially destroyed upon heating and bound water in this shell will be excluded to the bulk water. ${ }^{26}$ The hydrophobic $N$-isopropyl groups approach within their van der Waals radii and thus to form the hydrophobic bonding. If the solution is concentrated enough, the hydrophobic bonding between different polymer chains leads to a physically crosslinked network in the solution. In protein solutions, this hydrophobic bonding will cause the denaturation; e.g., the heat denaturation of $\beta$-lactoglubulin. ${ }^{27}$

According to the hydrophobic bonding mechanism, we gain some preliminary idea about the abnormal molecular weight dependence of cloud temperature in PNIPA/water solutions. The phase separation induced by the aggregation of hydrophobic alkane side chains is similar to the micell formation in a surfactant/water solution beyond the CMC (critical micell concentration). If these side chains were not linked by a backbone, there would be no either molecular weight or concentration dependence. Because the isopropyl groups are attached to the main chain of PNIPA, their aggregation to produce macroscopic turbidity will drag the polymer chain to move. This movement should overcome the energy barrier which may be higher for longer chains, resulting in the occurrence of phase separation at higher temperature for PNIPA having higher molecular weight. In thermodynamic terminology, this phase separation is mainly driven by the increase in enthalpy due to the change in the hydrated shell of the PNIPA chains. A suitable molecular weight dependence should be included in the enthalpy function for theoretical interpretation.

The problem here is how to determine the microgelation temperature $T_{\text {gel }}$. Winter et al..$^{28-30}$ proposed a method to determined the gel point for the chemical and physical gels, but their variable was the reaction (or gelation) time of the system at a constant temperature. Its feasibility for the microgelation in our PNIPA/water solutions induced by heating is still an open question. We recognize from Figures 5 through 9 that this transition temperature should be within $29-32.4^{\circ} \mathrm{C}$, lower than the corresponding cloud temperature $T_{\text {clo }}$ of $32.9^{\circ} \mathrm{C}$ for the D1/water solution. The difference may be attributed to the different aspects measured for the same behavior. The $T_{\text {clo }}$ reflects the occurrence of macroscopic turbidity while the $T_{\text {gel }}$ indicates the formation of microgels. Thus, it is reasonable that the change in the integral appearance of the solution occurs at the temperature higher than that in its microstructure.

Acknowledgments. The finacial supports to this work by the "Chinese National Science Foundation for Outstanding Young Scientists," the "Trans-Century Training Program Foundation for the Talents" by the State Education Commission of China, and the fund of the Higher Education Bureau of Guangdong Province are acknowledged gratefully.

\section{REFERENCES}

1. H. G. Schild, Prog. Polym. Sci., 17, 163 (1992).

2. K. Kubota, S. Fujishige, and I. Ando, J. Phys. Chem., 94, 5154 (1990).

3. S. Saeki, N. Kuwahara, S. Konno, and M. Kaneko, Macromolecules, 6, 246 (1973).

4. S. Saeki, S. Tsubotani, H. Kominami, M. Tsubokawa, and T. Yamaguchi, J. Polym. Sci., Polym. Phys. Ed., 24, 325 (1986).

5. I. Yamamoto, K. Iwasaki, and S. Hirotsu, J. Phys. Soc. Jpn., 58, 210 (1989).

6. S. Fujishige, K. Kubota, and I. Ando, J. Phys. Chem., 93, 3311 (1989).

7. C. Wu and S. Zhou, Macromolecules, 28, 5388 (1995).

8. C. Wu and S. Zhou, Macromolecules, 28, 8381 (1995).

9. F. M. Winnik, Polymer, 31, 2125 (1990).

10. F. M. Winnik, Macromolecules, 23, 233 (1990).

11. H. G. Schild and D. A. Tirrell, Macromolecules, 25, 4553 (1992).

12. H. Ringsdorf, J. Simon, and F. M. Winnik, Macromolecules, 25, 5353 (1992).

13. M. Heskins and J. E. Guillet, J. Macromol. Sci. Chem., A2, 1441 (1968).

14. H. G. Schild and D. A. Tirrell, J. Phys. Chem., 94, 4352 (1990).

15. K. Otake, H. Inomata, M. Konno, and S. Saito, Macromolecules, 23, 283 (1990).

16. F. Zeng, Z. Tong, and H. Feng, Polymer, 38, 5539 (1997).

17. F. Zeng, Z. Zheng, and Z. Tong, Polymer, in press.

18. X. Xie, F. Zeng, and Z. Tong, J. South China Univ. Tech. (in Chinese), in press.

19. O. Chiantore, M. Guaita, and L. Trossarelli, Macromol. Chem., 180, 969 (1979).

20. Z. Tong, J. South China Univ. Tech. (in Chinese), 18, 1 (1990).

21. R. Koningsveld, Adv. Colloid Interface Sci., 2, 151 (1968).

22. P. J. Flory, J. Am. Chem. Soc., 87, 1833 (1965).

23. D. Patterson, Macromolecules, 2, 672 (1969).

24. J. D. Ferry, "Viscoelastic Properties of Polymers," 3rd ed, John Wiley \& Sons, New York, N.Y., 1980.

25. M. Shibayama, Y. Suetoh, and S. Nomura, Macromolecules, 29, 6966 (1996).

26. A. K. Lele, M. M. Hirve, M. V. Badiger, and R. A. Mashelkar, Macromolecules, 30, 157 (1997).

27. P. Relkin, L. Eynard, and B. Launay, Thermochim. Acta, 204, 111 (1992).

28. H. H. Winter and F. Chambon, J. Rheol., 30, 367 (1986).

29. F. Chambon and H. H. Winter, J. Rheol., 31, 683 (1987).

30. W. Hess, T. A. Vilgis, and H. H. Winter, Macromolecules, 21, 2536 (1988). 